\title{
Joint SSRTNet/ALS-MES Workshop
}

This joint workshop brought together experimentalists and theorists interested in synchrotron radiation and highlighted subjects relevant to molecular environmental science (MES). The strong mutual interest between the participants resulted in joint sessions on the first day, followed by more specialized parallel sessions on the second day. Held in conjunction with the Advanced Light Source (ALS) Users' Association Annual Meeting at the Lawrence Berkeley National Laboratory (Berkeley Lab), the Synchrotron Radiation Research Theory Network (SRRTNet) workshop was co-organized by Michel Van Hove (Berkeley Lab and University of California, Davis) and Andrew Canning (Berkeley Lab), while David Shuh (Berkeley Lab) organized the ALS-MES workshop.

SRRTNet is a global network that promotes the interaction of theory and experiment (http://www.cse.clrc.ac.uk/Activity/SRRTnet). The ALS-MES project is constructing Beamline 11.0.2.1-2, a new soft $\mathrm{x}$-ray beamline for MES investigations at photon energies from $75 \mathrm{eV}$ to 2 $\mathrm{keV}$, to provide photons for wet spectroscopy end stations and an upgraded scanning transmission $\mathrm{x}$-ray microscope (STXM). The ALS-MES beamline and end stations will be available for users in the late fall of 2002.

The workshop opened with a plenary presentation by Gordon Brown, Jr. (Stanford University), who stressed the importance of obtaining solid information to form the basis for an improved understanding of fundamental chemical processes in complex environmental systems. Recent results were presented that illustrated the utility of soft x-ray investigations, including photoemission and NEXAFS measurements, from several key environmental interfaces, including that between water and solids. Prospects for new MES studies utilizing the ALS-MES beamline and the importance of the resulting improved scientific knowledge were also discussed.

Nicholas Harrison (Imperial College London and CLRC Daresbury Laboratory) described hybridexchange density functionals for the prediction of structure, energetics, and excited states at interfaces. Such functionals in periodic calculations of solids have significantly increased the accuracy of ground-state energy surfaces and also the ability to predict bulk and surface electronic structure. The interaction between simulation and experiment based on these advances has been very fruitful.

Adam Hitchcock (McMaster University) covered recent results from STXM investigations utilizing silicon nitride cells to study wet water-filtration membranes, protein adsorption on polymers, and metal bio-accumulation by biofilm systems. Of particular interest, the biological components of the biofilm (protein, lipid, saccharide, DNA) were quantitatively mapped, and the distribution of $\mathrm{Ni}^{2+}$ was also mapped relative to these biofilm components. In addition, new data analysis techniques for STXM were described. 
Jim Rustad (Pacific Northwest National Laboratory) discussed recent progress made in the simulation of aqueous interfacial systems with limited degrees of freedom and compared them with real-life interfaces of interest today, which have tremendous complexity. Several examples involving important aqueous interfaces were presented in which simulation was able to resolve inconclusive experimental results and, in some cases, suggested novel interpretations of the results.

Miquel Salmeron (Berkeley Lab) presented the latest accomplishments obtained with the differentially-pumped vacuum system housing the high-pressure electron-transfer system (HPETS) at ALS beamline 9.3.2, which enables photoemission and NEXAFS investigations of surfaces in thermodynamic equilibrium with gas species at pressures up to a few Torr. He discussed the premelting/melting transitions of the ice surface and the methanol-to-methanal conversion over copper catalyst with simultaneous detection of both reactants and reaction products in the electron lens nozzle manifold.

John Rehr (University of Washington, Seattle) reviewed progress and challenges in soft x-ray absorption spectroscopy (XAS). There has been dramatic progress over the past decade both in theory and ab initio calculations of XAS spectra, particularly for deep-core XAS. Theories of soft x-ray XAS are more challenging but have recently yielded excellent results, as reflected in a number of recent FEFF code upgrades.

Anders Nilsson (Stanford University) described investigations using x-ray spectroscopy and theory to study the electronic states for two uniquely important types of chemical bonding; the surface chemical bond in molecules adsorbed on surfaces and the hydrogen bond in water and ice. The results showed the unique character of the electronic structure of liquid water, suggesting that specific configurations of water exist for which the hydrogen-bond is broken on the huydrogen-donating site of the water molecule.

Scott Chambers (Pacific Northwest National Laboratory) communicated the findings and implications from an investigation of the effects of calcium carbonate coatings on the adsorption of aqueous $\mathrm{CrO}_{4}{ }^{2-}$ onto epitaxial $\mathrm{Fe}_{3} \mathrm{O}_{4} / \mathrm{MgO}(001)$ surfaces, using high-energy-resolution x-ray photoemission spectroscopy (XPS) in conjunction with scanning-probe microscopy. One important theme was the novel preparation of the $\mathrm{CaCO}_{3}$ coating phases. The effects and environmental importance of carbonate coatings on substrate reactivity were discussed in particular.

Glenn Waychunas (Berkeley Lab) introduced the areas of environmental nanotechnology particularly well-addressed with x-ray spectroscopy: the characterization of inherently small and highly defective natural nanoparticulates with unique reactive properties (such as the iron oxyhydroxide sulfate schwertmannite), the elucidation of formation mechanisms and structure of initially formed nanoprecipitates on mineral surfaces (exemplified by zinc complexation on ferrihydrite surfaces), and identification of the nanoscale aggregation processes that lead to 
polytype formation, grain-boundary sequestration of impurities, and dramatic alteration of transport behavior in the environment.

Chuck Fadley (Berkeley Lab and University of California, Davis) presented several examples that highlighted emerging directions for investigating nanostructures and nanometer-scale heterogeneity with x-ray spectroscopies and scattering experiments. The examples, from both soft and hard x-ray regions, paid special attention to theoretical developments necessary for the proper interpretation of the experimental results.

The second day of the workshop, running as parallel sessions, saw the SRRTNet participants focus more on the theoretical understanding of x-ray and electron scattering measurements, as well as comparing predictions from ab initio calculations with SR experiments. George Sawatzky (University of British Columbia) addressed the modeling of resonant x-ray scattering, emphasizing that in most cases band structure effects are very important. He also presented new results on resonant scattering in systems without inversion symmetry.

Paolo Carra (European Synchrotron Radiation Facility) presented his theory of the new technique of non-reciprocal $\mathrm{x}$-ray dichroism in $\mathrm{V}_{2} \mathrm{O}_{3}$, specifically for photo-absorption spectroscopies in non-centrosymmetric systems, covering both $\mathrm{x}$-ray and optical regions. Symmetry arguments afford a classification of valence-electron states in the presence of parity non-conserving hybridization.

Kai Starke (Freie Universit t, Berlin) considered the magneto-optical Kerr effect in the x-ray regime of rare-earth systems. He demonstrated the feasibility of element-specific magnetization reversal studies by applying the x-ray magneto-optical Kerr effect (XMOKE) to lanthanide systems.

Norikazu Tomita (Photon Factory, Tsukuba) presented a non-Grassmann path integral theory to calculate momentum-specified photoemission and light absorption spectra of many-electron systems with strong long-range Coulomb repulsion. The calculations were successfully compared with experiment for one- and two-dimensional spin-density wave states of $\mathrm{Ni}-\mathrm{Br}$ and $\mathrm{Cu}-\mathrm{O}$ compounds

Kenji Makoshi (Himeji Institute of Technology and SPring8) discussed recent work on the magnetic Compton profile (MCP) of bilayer manganite $\mathrm{La}_{(2-2 \mathrm{x})} \mathrm{Sr}_{(1+2 \mathrm{x})} \mathrm{Mn}_{2} \mathrm{O}_{7}$. Experimental results for this material were compared with theoretical calculations of $\left(\mathrm{MnO}_{6}\right)^{8-}$ and $\left(\mathrm{MnO}_{6}\right)^{9-}$ clusters. Among other results, he confirmed that the MCP detects the oxygen hybridization in the $\mathrm{e}_{\mathrm{g}}$ orbitals.

O. Sipr (Academy of Sciences, Prague) addressed the question "Where is the XANES photoelectron?" with an exploration of wave function probability densities. He compared the probabilities that the XANES photoelectron with a given energy can be found in the vicinity of individual atoms. 
Hubert Ebert (Universit t M nchen) presented a theoretical description of spin-polarized angleintegrated photoemission of para- and ferromagnetic solids, based on the one-step model of photoemission and relativistic multiple-scattering theory. Applications to valence-band photoemission from copper, silver, and gold led to very satisfying agreement with recent experiments.

Van Hove covered recent progress in the theory of photoelectron diffraction and holography, in order to achieve wider applicability, greater performance and higher accuracy. Included were electron-spin effects, photon polarization, non-spherical scattering potentials, and a new technique of differential photoelectron holography.

Lin-Wang Wang (Berkeley Lab) described the development of a charge-patching method to generate the LDA charge density of a large system without doing an actual self-consistent calculation for the system, by combining simple "motifs" to produce the charge density of the large system.

Warren Pickett (University of California, Davis) brought the audience up to date on the understanding of the newly discovered superconductivity in $\mathrm{MgB}_{2}$, and discussed the potential role of unusual collective charge excitations in this material. . Theory strongly suggests that superconductivity in $\mathrm{MgB}_{2}$ is driven by aspects of the structure that result in strongly covalent bonds becoming metallic, creating very strong coupling to bond stretching modes.

The second day of the workshop saw the ALS-MES participants address more specific scientific issues related to the first experiments planned for the beamline, the capabilities needed for these experiments, and the status of the beamline construction project. Shuh and Tony Warwick (ALS) described the progress to date on several aspects of ALS-MES Phase I Project. These updates included a general report on the status of the overall project and the progress being made with respect to the construction of the Phase I beamline. These brief project reports were followed by a current description of the end stations and the status of end-station activities. There also was a substantial period of open discussion with the ALS-MES participants concerning issues germane to the scientific programs that will employ the wet spectroscopy end stations and to the recently implemented STXM upgrade.

Brief scientific presentations followed from ALS-MES Steering Committee members, potential new users, and scientists interested in exploiting the unique capabilities of the beamline/end stations for experiments early in the operational tenure of the new beamline. These more detailed scientific talks focused on specific scientific opportunities, experimental requirements, and pertinent recent developments.

The first speaker was Frank Ogletree (Berkeley Lab), who described the opportunities in several scientific areas that will become available when the second-generation ambient-pressure photoemission spectrometer is implemented on the ALS-MES Phase I beamline. The current 
design of the spectrometer and preparation system was presented. The operational parameters and the anticipated performance characteristics of the end station were discussed. Translating these technical specifications into science, it was clear that this end station will provide new, unique opportunities for MES and for other fields such as catalysis. Several classes of possible experiments using the end station were presented.

Nilsson described the importance of new studies that will be possible with the ALS-MES beamline based on the results of similar experiments to date, primarily focusing on future investigations involving water and small, complex organic molecules at surfaces.

Jim Tobin (Lawrence Livermore National Laboratory) discussed recent progress made by the collaborative national laboratory research team (Livermore, Berkeley Lab, and Los Alamos National Laboratory) investigating actinide materials by photoelectron spectroscopy at the ALS, with an emphasis on the importance of and the possibilities in this area in the near future. Highlighted were the unique attributes of the ALS for performing resonant photoemission and high-resolution core-level spectroscopy, as well as the ability to perform such measurements on very small amounts of radioactive material.

Shuh briefly continued the theme of investigating actinide electronic structure by use of x-ray emission and absorption in the fluorescence mode to avoid sample preparation complications. Results from resonant inelastic x-ray scattering measurements of the uranium oxides and the correspondence to calculated spectra were shown.

Jeff Kortright (Berkeley Lab) spoke about several of the unique opportunities to investigate magnetic materials arising from the first combination of STXM with an elliptically-polarized undulator. Of primary importance will be the increased intensity since there will be no need for a polarizing element, which translates to a minimum ten-fold flux enhancement plus higher sensitivity to ferromagnetism.

\author{
Michel Van Hove \\ Materials Sciences Division \\ and \\ David Shuh \\ Chemical Sciences Division \\ Berkeley Lab
}




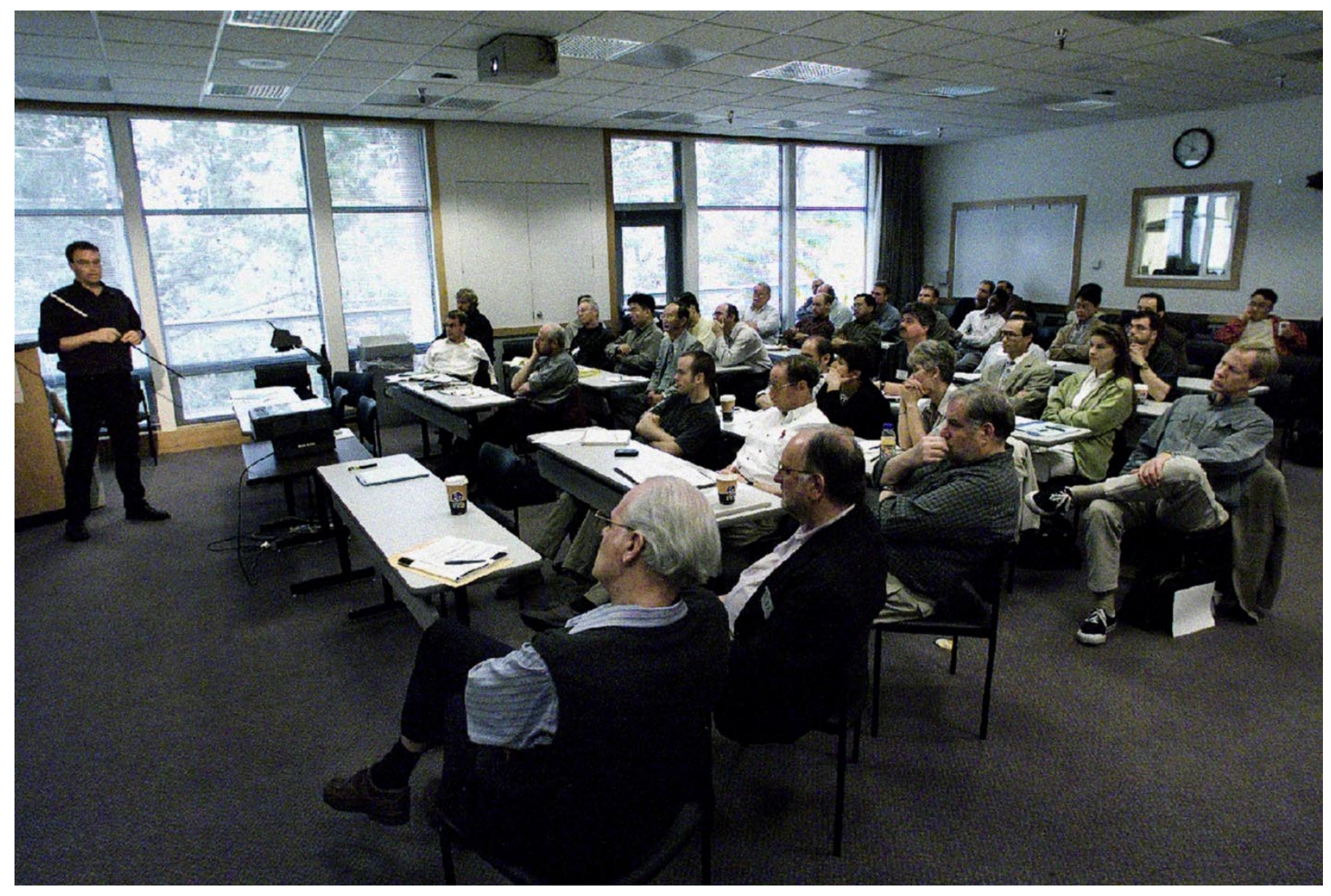

Nicholas Harrison answers questions at the conclusion of his SRRTNet/ALS-MES Workshop presentation in Berkeley Lab's Perseverance Hall.

This work was supported by the Director, Office of Science, Office of Basic Energy Sciences, Chemical Sciences, Geosciences, and Biosciences Division and the Materials Sciences Division of the U. ${ }^{\circ}$ S. Department of Energy (DOE) under Contract No. DE-AC03-76SF00098 at LBNL. 